\title{
Erratum
}

\section{Erratum. APPL1 Counteracts Obesity-Induced Vascular Insulin Resistance and Endothelial Dysfunction by Modulating the Endothelial Production of Nitric Oxide and Endothelin-1 in Mice. Diabetes 2011;60:3044-3054 \\ DOI: $10.2337 / d b 16$-er10c}

In the article listed above, an erroneous duplication was made during the process of image assembly for total ERK1/2 in Fig. $4 A$. Lanes 4 and 5 in the original experiment were duplicated by mistake when loading the samples during SDS-PAGE. In order to remove lane 5 for a unified presentation in Fig. $4 A$, the authors created a new file to assemble the image by copying and pasting the first four lanes in the STD group and the last four lanes in the HFD group. However, the last four lanes were copied twice by mistake. The right panel in this revised version shows the correctly assembled image for total ERK1/2 from the original data, which is shown in the left panel. This error was unintentional. In addition to total ERK1/2, total Akt and $\alpha$-actin are appropriate as loading controls for this experiment. The authors apologize to the readers for any confusion.
Yi Wang, Kenneth K.Y. Cheng, Karen S.L. Lam, Donghai Wu, Yu Wang, Yu Huang, Paul M. Vanhoutte, Gary Sweeney, Yiming Li, and Aimin Xu

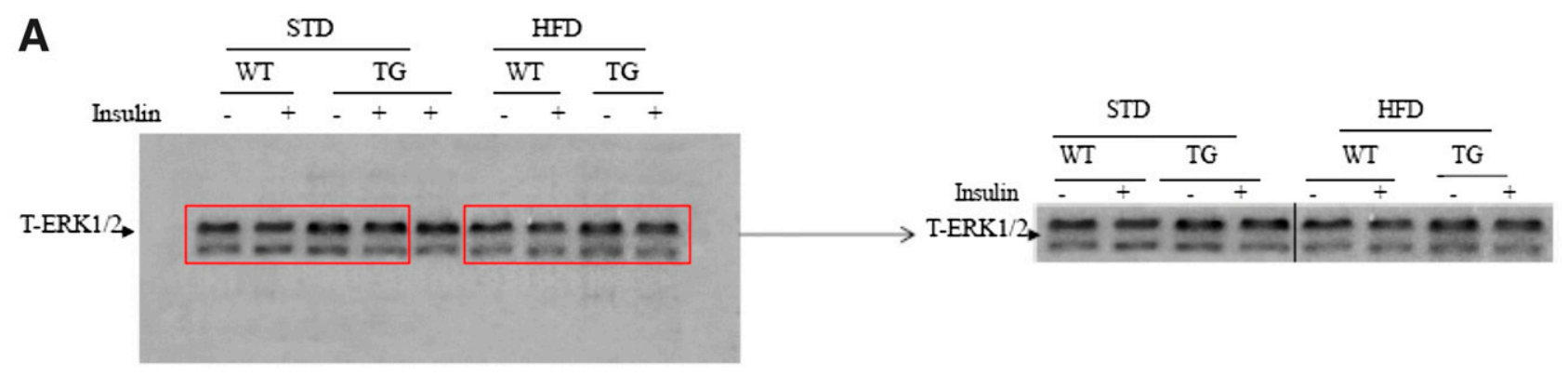

